\title{
Virtual Reality and Desktop as a Combined Interaction-Visualisation Medium for a Problem-Solving Environment
}

\author{
E.V. Zudilova and P.M.A. Sloot \\ Section Computational Science \\ Faculty of Science, University of Amsterdam \\ Kruislaan 403, 1098 SJ Amsterdam, The Netherlands \\ [elenaz|sloot] @science.uva.nl
}

\begin{abstract}
The paper addresses the problem of how to make a human-machine interaction user-friendlier within a problem-solving environment. Two different projection modalities - virtual reality and desktop solution - are compared in respect to interaction capabilities provided by the Virtual Radiology Explorer (VRE) - case study of this research. The VRE is a problem-solving environment for vascular reconstruction, developed by the Section Computational Science of the University of Amsterdam. The potential users of the VRE are physicians, whose attitudes and motivations vary. The combination of virtual and desktop interaction modes within the same environment may help to satisfy the wider range of VRE users, in comparison to the case when only one projection modality is used. A Personal Space Station is considered as a possible solution for deploying this concept.
\end{abstract}

\section{Introduction}

A problem-solving environment (PSE) provides a primary (or end) user with a set of hardware and software resources for building a specific framework to solve a target class of problems. Ideally this framework is to be built in such a way that a user can exploit modern technologies without specialised knowledge of underlying hardware and software.

In reality the situation is far from ideal. It is supposed that a primary user knows how to use simulation and visualisation programs (libraries, modules, software components, etc.) and that he is able to characterise a problem to be solved using a specific definition language. Most of developers do not take into account the fact that a primary user of a PSE is a scientist, who focuses mostly on his research area, and he is not very experienced as a computer user and needs intuitive interaction capabilities and a feedback adapted to his skills and knowledge.

PSEs' developers focus today more and more on modern advanced technologies concerning also the projection equipment used as an interaction-visualisation 
medium. The choice of projection equipment today is mostly task-related or even spontaneous. As a result, a human-machine interaction provided by PSEs is far from intuitive. Existing projection modalities have not been investigated yet in respect to usability factors. Meanwhile, the selection of an appropriate projection modality in accordance with user's tasks, preferences and personal features has to be used as a basis for building a motivated PSE.

The PSE introduced in this paper is a framework for rapid prototyping of an exploration environment that permits a user to explore interactively the visualised results of a simulation and manipulate the simulation parameters in near real-time, where a pre-operative planning of a vascular reconstruction procedure is a test case for making experiments.

Section 2 of the paper contains the description of the Virtual Radiology Explorer (VRE): its functionality, architecture and main interaction capabilities. Section 3 classifies users of the VRE and their needs in respect to different projection modalities. In section 4 two different approaches of deploying interactionvisualisation capabilities within a PSE are presented. The Virtual Operating Theatre and the Individual Desktop Environment are differentiated by functional and interaction capabilities needed by different groups of users of the VRE. The notion of a Personal Space Station is introduced as a possible solution to combine two these mediums within the same environment.

\section{The Simulated Environment for Vascular Reconstruction}

\subsection{Introduction to the Vascular Reconstruction}

Vascular diseases affect arteries and veins. Vascular disorders in general fall into two categories: aneurisms and stenosis. An aneurysmal disease is a balloon like swelling in the artery. Stenosis is a narrowing or blockage of the artery. The purpose of the vascular reconstruction is to redirect and increase blood flow or repair a weakened or aneurysmal artery if necessary.

There are several imaging techniques that can be used to detect vascular disorders. 3D data acquired by computed tomography (CT) or magnetic resonance imaging (MRI) is converted into a set of $2 \mathrm{D}$ slices that can be displayed and evaluated from various perspectives and at different levels. Magnetic resonance angiography (MRA) is a technique for imaging blood vessels that contain flowing blood. It is very popular among cardiovascular specialists because of its ability to non-invasively visualise a vascular disease.

The verification of the operation plan is one of the most complicated tasks in vascular surgery. Different treatments for vascular diseases exist today. They include adding shunts and bypasses in the case of aneurysms and applying thrombolysis techniques, balloon angioplasty, bypasses and stent placement for a stenosis. The best treatment is not always obvious because of the complexity of a vascular disease and because of another diseases that a patient may have. 


\subsection{A Design Concept}

The Virtual Radiology Explorer (VRE) gives a possibility to verify whether the selected treatment is the best in the current circumstances.

The VRE is a PSE, that puts a user into an experimental cycle simulated by a computer and let him apply his expertise to find better solution for the treatment of a vascular disease. The criterion of the success of a treatment is the normalisation of a blood flow in the affected area. The procedure of adding a bypass is of the most interest to us as it can be used both for treatment of aneurysms and stenosis.

The design concept of the VRE is represented on Fig.1.

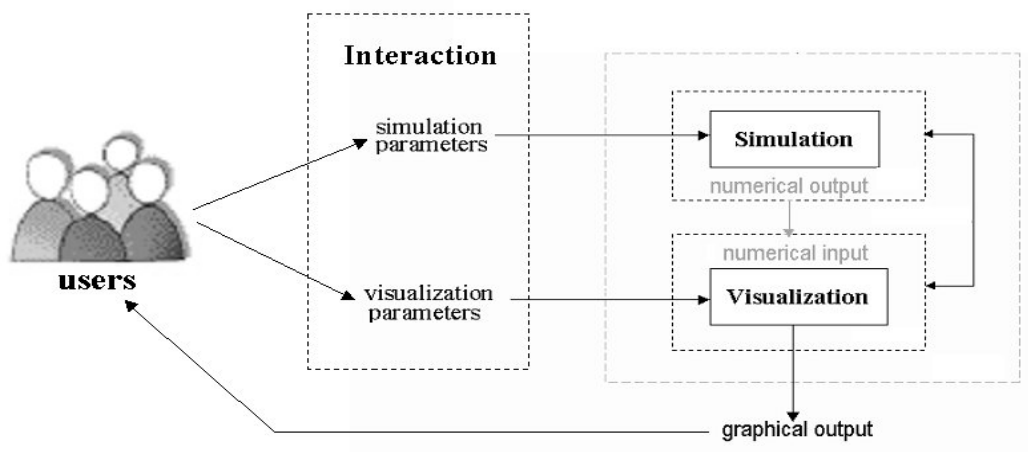

Fig. 1. A design concept

A simulation part of the VRE simulates the parameters of a patient's blood flow, i.e. velocity, pressure and shear stress. A user monitors the simulation process and controls the blood flow parameters. He has a possibility to add a bypass and to check whether a blood flow was normalised or not. He can also change the visualisation parameters, such as sample rate, scale, colour palette, light effects, etc.

The input for conducting experiments is scanned data of a patient stored in a database, which also contains an archive of interesting cases and interesting vascular images. This information is useful for both diagnostics and planning a treatment.

Fig.2 represents the current architecture of the VRE system, where the starting point is a scanner, and the front-end to the system is the Distributed Real-time Interactive Virtual Environment (DRIVE) system - a PC based environment, including a single-wall projection device developed by our group in the University of Amsterdam [1, 12].

The concept of interaction in virtual reality was selected as a basis for building the DRIVE system. Stereovision is the normal way almost everyone sees in the real world [4]. The virtual reality permits to build an environment where users interact freely with a $3 \mathrm{D}$ space and entities within it. 
The working prototype of the VRE is provided with a multi-modal interface described in [10]. It combines natural input modes of context sensitive interaction by voice, hand gestures and direct manipulation of virtual 3D objects. We called this interaction mode the 'Virtual Operating Theatre', as a user 'plays' a role of a vascular surgeon applying a treatment of a vascular disease on a simulated patient [2].

The functionality of the VRE has been described in earlier publications $[2,3,10]$. Data conversion, segmentation, LBM-grid [5] generation, fluid flow simulation, surface and volume rendering are complicated computational tasks that need additional resources. But they are not very interesting in respect to human-machine interaction, as most of them are non-interactive or just can be run, paused or stopped by a user.

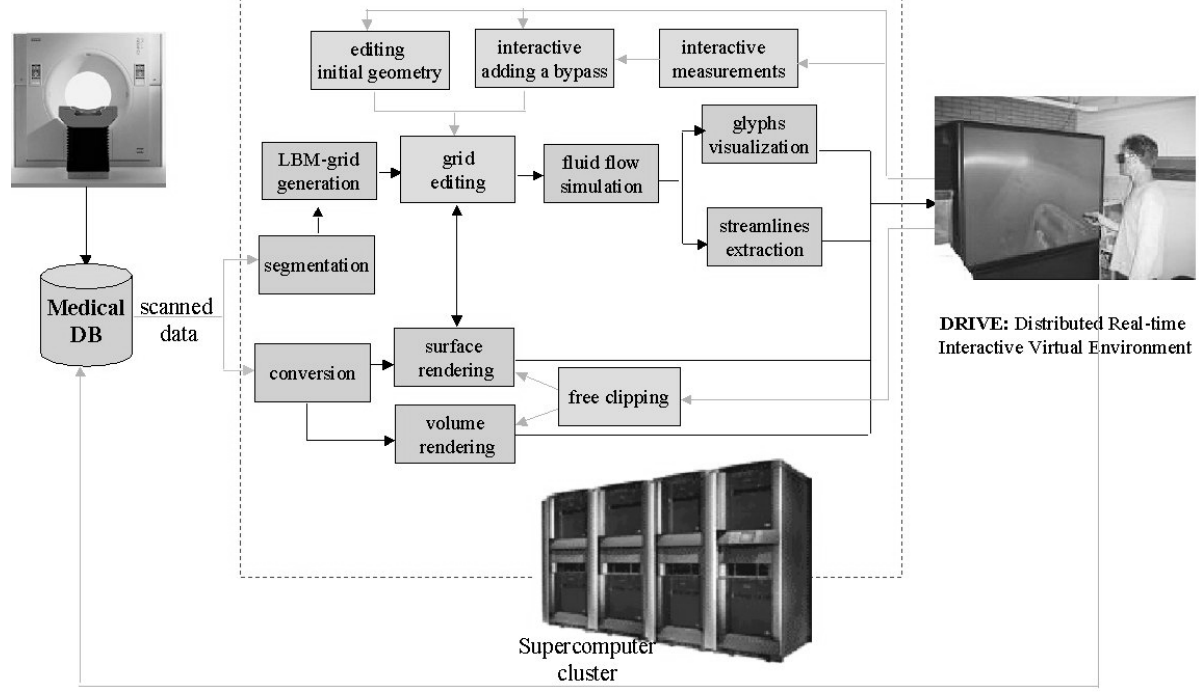

Fig. 2. Experimental set-up for the vascular reconstruction

\subsection{Interaction Capabilities}

Human-machine interaction within the VRE is concentrated mainly on retrieving data about a patient from a Medical DataBase (DB), conducting interactive measurements and grid editing.

Grid editing provides the possibility to edit the initial geometry of an artery: to remove insignificant elements or restore the fragments lost during the segmentation. Grid editing permits a user to create a bypass and place it on an artery. A bypass is a graft rerouting a blood flow around blockages. Usually it is a piece of vein taken from elsewhere in the body or an implant made from an organic material.

Measurements are important for diagnostics. Clinical decision-making relies on evaluation of the vessels in terms of a degree of narrowing for stenosis and dilatation 
(increase over normal arterial diameter) for aneurysm. The selection of a correct bypass (its shape, length, diameter) also depends on sizes and geometry of an artery. The interactive measurement component of the VRE [3] provides the possibility to measure quantitatively a distance, angle, diameter and some other parameters characterizing a 3D object in a virtual world. For conducting a measurement, a user has to position a corresponding number of active markers on an object.

A free clipping engine, which we are developing currently, is also an interactive component of the VRE. It permits to build clipping planes of different orientations in a 3D space. Having made a section of a 3D object by means of a clipping plane, a user can look inside an artery. Moving a clipping plane he will see the original data obtained from a scanner slice by slice.

\section{User Attitudes to Different Projection Modalities}

\subsection{Users}

The VRE users are divided into two main groups (see Fig. 3):

- $\quad$ System managers;

- Primary users.

A system manager is responsible for maintenance and support of primary users, local software and hardware availability, remote data transfer and management of distributed computational resources. A system manager deals with system and

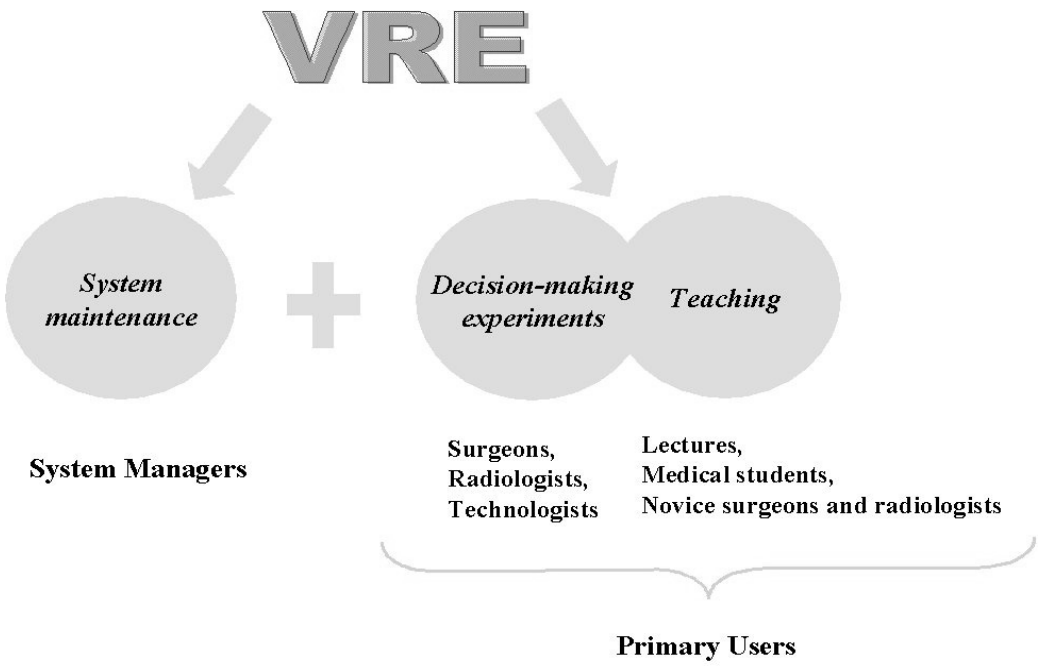

Fig. 3. User Classification 
resource management: reliable network connection, secure authentication of primary users, access to computational-visualisation resources, availability of staff for specific equipment (MRA/MRI/CT scanners), etc.

Primary users are people, who use the VRE as a tool for conducting experiments. It is expected that the VRE will be used for the interactive decision support by vascular surgeons, radiologists (both diagnosis and interventional) and technologists. Vascular technologists are people from scientific or radiography background. They conduct patients' testing using special equipment, including MRA/MRI/CT scanners, for diagnosis of arterial and venous diseases. The VRE can also be used for training of medical students and novice physicians.

Potential users of the VRE differentiate by their preferences, skills, motivation, cognitive features and stress factors [11]. As a result, their expectations about the interaction within the VRE also vary.

User comfort is very important for the success of any software application. The working prototype of the VRE is almost finished. That's why we focus our today's efforts mostly on the improvement of the interaction and visualisation capabilities within the VRE. The primary users are of the most interest to us because most of them are inexperienced computer users and they expect that a PSE will provide a humanmachine interaction similar to the interaction in a real world.

\subsection{Users' Needs: Virtual Operating Theatre or Personal Desktop Environment?}

A human-machine interaction depends to a great extent on projection equipment selected for deploying an interaction-visualisation medium. Today's existing solutions differentiate by information and visual design, provided forms of navigation, locomotion, selection and manipulation.

The research presented in this paper is focused on comparison of two projection modalities: the Virtual Operating Theatre introduced in section 2 and Personal Desktop Environment, which is an interaction-visualisation mode supported by standard PC/PDA applications.

The heuristic evaluation [6] of the VRE and the first user interviews, conducted recently, show that the concept of the Virtual Operating Theatre does not satisfy all VRE users.

It was already mentioned above that the DRIVE system was selected as projection equipment for deploying a concept of the Virtual Operating Theatre. One of the challenges of the DRIVE system is that it provides a shared interaction environment [12].

'One-to-one' semi-structured interview, conducted recently to identify the important variables for user profiling, shows that there is a possibility that some surgeons and interventional radiologists will prefer to use a personal desktop version of the exploration environment for accomplishing every-day tasks. As for the shared environment, it may happen, that it will be used only for the collaborative work, i.e. for training or medical conferences when the diagnosis and possible treatments are discussed within a group of people. Both hypotheses will be checked via series of 
semi-structured interviews. The preferences of technologists and diagnosis radiologists with respect to different projection modalities are not obvious currently and are also part of future analyses.

Like it was mentioned above, the VRE system can be used as a training environment for novice physicians.

Two modes of interactive training exist [11]:

- $\quad$ Lecture mode;

- Tutorial mode.

If it is a 'lecture mode', a lecturer, responsible for all training components, including theoretical, demonstrational and practical parts of a course, guides a class. In this case the concept of the Virtual Operating Theatre is a good solution accepted under the condition that the number of students in a group is optimal. A 'tutorial mode' is oriented to students who want to study material independently. The individual environment will satisfy them most of all. At the moment the VRE can be used only in a lecture mode because the concept of the Virtual Operating Theatre does not fit well to a tutorial mode of interactive training.

Such factors as users' preferences and motivation, discussed above, have an influence on the selection of an appropriate projection modality. But there exist one more factor, which is the most important in this respect. This factor is called 'simulator sickness'.

A simulator sickness $[4,9]$ is a kind of motion sickness except that it occurs in a simulated environment without actual physical motion. The simulator sickness occurs in conjunction with the virtual reality exposure. Users having simulator sickness cannot work in a virtual reality. According to [9] almost a quarter of computer users have simulator sickness. So approximately the same proportion of the VRE users will be unable to work with its virtual environment. For this type of users desktop remains the only possible solution.

\section{A Personal Space Station as a Combined Interaction Medium}

A Personal Space Station (PSS) is a relatively new concept for the implementation of an interaction-visualisation environment that may help to solve problems mentioned above [4].

The goal of a PSS is to allow a user to interact directly with a virtual world. A PSS consists of a mirror in which a stereoscopic image is reflected. The user reaches under the mirror to interact with the virtual objects directly with his hands or by using taskspecific input devices. The main advantage of a PSS is that it combines both elements of desktop and virtual projection modalities within the same environment. And it is possible to switch in between if necessary.

A PSS is an individual environment by definition, but there is a possibility to build a shared environment where users can manipulate the same virtual objects working on different PSSs. More information about the PSS concept can be found in [7]. 


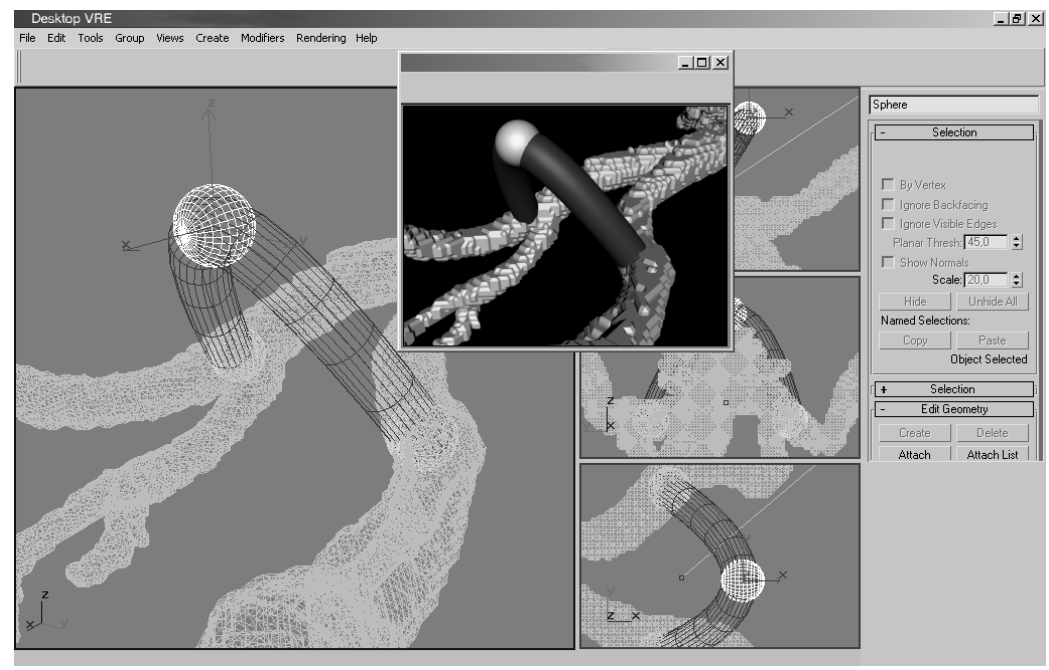

Fig. 4. Grid-editing tool of the desktop version of the VRE

The idea to combine virtual and desktop projection modalities within the same interaction-visualisation medium looks very attractive. In this case different types of users will be able to work within the same environment having a minimal feeling of discomfort. But building such combined medium is not an easy task. First of all, we have to take into account, that interaction in virtual and desktop projection modalities is different in respect to navigation, locomotion and manipulation capabilities $[4,8$, 9]. To provide the possibility to switch between virtual and desktop interaction modes within the VRE, its current interface has to be changed significantly, as it is oriented currently mostly on the interaction in a virtual reality.

Let us compare a procedure of adding a bypass in virtual and desktop projection modalities. In a virtual environment a user manipulates $3 \mathrm{D}$ objects. He deals with $3 \mathrm{D}$ representations of an artery and a bypass. For building a bypass within the VRE a spline primitive is used. The procedure of adding a bypass in a virtual environment comes down to re-scaling of a spline and its correct positioning on an artery. For these manipulations a user has a wand. To manipulate successfully in a $3 \mathrm{D}$ virtual world a user should possess some special motor skills of navigation and manipulation, which sometimes are not trivial and depend on the level of implemented interaction capabilities.

As for the interaction in a desktop environment, user does not need additional motor skills. The main problem here is that within desktop applications we cannot manipulate 3D objects directly, we always deal with $2 \mathrm{D}$ projected representations of these objects [9]. Fig. 4 presents a mock-up of the graphical editor for adding a bypass within a desktop version of the VRE. 3D representation of objects does not have a big sense for a desktop environment. It is just a passive viewer, while a user adds a bypass operating with several projections of an artery, which orientations are predefined manually. 
The same situation we have with a clipping procedure. In the case of virtual reality a user can change orientation of a clipping plane using a wand. He can navigate in a virtual world, look inside an artery and even walk through. In a desktop version additional interface capabilities have to be applied. For instance, a user can select slices of interest be means of a menu or a slider. A unique identification number will help to define a concrete slice.

As for interactive measurements, like it was already mentioned in section 2, a user first has to add active markers. Markers are building blocks of distance, angle and linestrip measurements [3]. The number of necessary active markers depends on a measurement to be done. For measuring a distance, a user has to add 2 markers, if it is angle -3 , for conducting linestrip or tracing measurements - at least 2. At the moment the interactive measurement component is available only for the virtual version of the VRE. Switching to a desktop projection modality leads to a necessity of deploying extra menus and sliders to simplify the work of a user in a projected 3D world.

\section{Discussion and Future Work}

In this paper a PSE for simulated vascular reconstruction has been introduced. The work is still in progress and one of the crucial issues to focus on is the improvement of interaction capabilities within it. The heuristic evaluation conducted recently and the initial stage of user profiling show that the human-machine aspects depend to a great extent on a projection modality selected for deploying an interactionvisualisation medium.

As the primary users of the VRE are physicians who are usually not very familiar with modern computer technologies, it is very important to make the process of their interaction within a PSE as much comfortable as possible.

Two concepts - the Virtual Operating Theatre and the Individual Desktop Environment - have been presented. It has been shown that both virtual reality and desktop solution are claimed by the VRE users. That's why we decided to combine virtual and desktop interaction capabilities within one medium. A PSS may help to bring this idea to life. Its main advantage is that it permits to switch from one to another projection modality if necessary. The experimental version of a PSS is currently being built in the University of Amsterdam within the Token 2000 project "Distributed Interactive Medical Exploratory for 3D Medical Images".

At the same time we are deploying a desktop version of a grid editing tool and free clipping engine. It is also planned to build a desktop version of an interactive measurement component of the VRE.

The final goal of this research is to investigate navigation, locomotion, manipulation and measurement capabilities within the Virtual Operating Theatre and the Individual Desktop Environment. The VRE running on a PSS will be an experimental environment for this research. Of course, of most interest to us are the attitudes of real users exploiting the VRE in their daily activities. For these case studies we will collaborate with the Amsterdam Medical Centre, the Leiden University Medical Centre and the Rotterdam Medical Centre. 
Acknowledgements. The authors would like to thank Robert Belleman, Roman Shulakov, Hans Ragas Denis Shamonin and Daniela Gavidia (Section Computational Science, UvA) for the contribution to the development of the VRE; and Henriette Cramer and Dr. Vanessa Evers (Social Informatics Department, UvA) for the input to the usability studies.

\section{References}

1. Belleman R.G., Stolk B., de Vries R.: Immersive Virtual Reality on commodity hardware, Proceedings of the 7th annual conference of the Advanced School for Computing and Imaging, pp. 297-304. ASCI, May 2001 (2001).

2. Belleman R.G., Sloot. P.M.A.: Simulated Vascular Reconstruction in a Virtual Operating Theatre. CARS 2001 Conference (CARS2001), Berlin, Germany, June 2001 (2001).

3. Belleman R.G., Kaandorp J.A., Dijkman D., Sloot P.M.A: GEOPROVE: Geometric Probes for Virtual Environments, Proc. of HPCN Europe '99, Amsterdam, The Netherlands, pp. 817-827 (1999).

4. Bowman D.G., Hodges L.F.: User Interface Constraints for Immersive Virtual Environment Applications. Graphics, Visualization and Usability Center Technical Report GIT-GVU-95-26 (1995).

5. Kandhai B.D., Koponen A., Hoekstra A.G., Kataja M., Timonen J. and Sloot P.M.A.: Lattice Boltzmann Hydrodynamics on Parallel Systems, Computer Physics Communications, vol. 111, pp. 14-26 (1998).

6. Nielsen J.: Usability Engineering, Academic Press (2000).

7. Mulder J.D., van Liere R.: The Personal Space Station: Bringing Interaction within reach. Center for Mathematics and Computer Science, CWI, Amsterdam, The Netherlands (2001) http://www.cwi.nl/ robertl/papers/2002/laval/.

8. Pierce J., Forsberg A., Conway M.J., Hong S., Zeleznik R.: Image Plane Interaction Techniques in 3D Immersive Environments, Proceedings of 1997 Symposium on Interactive 3D Graphics, pp. 39-43 (1997).

9. Raskin J.: The Humane Interface: New Directions for Designing Interactive Systems, Addison-Wesley Pub Co (2000).

10. Zudilova E.V., Sloot P.M.A., Belleman R.G.: A Multi-modal Interface for an Interactive Simulated Vascular Reconstruction System, Proc. of the IEEE International Conference on Multimodal Interfaces, Pittsburgh, Pennsylvania, USA, October 2002, pp. 313-319 (2002).

11. Zudilova E.V., Sloot P.M.A.: A First Step to a User-Centered Approach to a Development of Adaptive Simulation- Visualization Complexes, Proc. of the International Conference of the Systemics, Cybernetics and Informatics, Orlando, Florida, USA, July 2002, V. V, pp. 104-110 (2002).

12. The University of Amsterdam Distributed Real-time Interactive Virtual Environment (UvA-DRIVE) webpage. http://www.science.uva.nl/ robbel/DRIVE/ 